\title{
Effect of the next-nearest-neighbor hopping on the charge collec- tive modes in the paramagnetic phase of the Hubbard model
}

\author{
Vu Hung DaO ${ }^{1}$ and RAYMond Frésard ${ }^{1}$ \\ 1 Normandie Univ, ENSICAEN, UNICAEN, CNRS, CRISMAT, 14000 Caen, France \\ PACS 71.10.Fd - Lattice fermion models \\ PACS 72.15. $\mathrm{Nj}$ - Collective modes \\ PACS $71.30 .+\mathrm{h}-$ Metal-insulator transitions and other electronic transitions
}

\begin{abstract}
The charge dynamical response function of the $t-t^{\prime}-U$ Hubbard model is investigated on the square lattice in the thermodynamical limit. The correlation function is calculated from Gaussian fluctuations around the paramagnetic saddle-point within the Kotliar and Ruckenstein slave-boson representation. The next-nearest-neighbor hopping only slightly affects the renormalization of the quasiparticle mass. In contrast a negative $t^{\prime} / t$ notably decreases (increases) their velocity, and hence the zero-sound velocity, at positive (negative) doping. For low (high) density $n \lesssim 0.5$ ( $n \gtrsim 1.5$ ) we find that it enhances (reduces) the damping of the zero-sound mode. Furthermore it softens (hardens) the upper-Hubbard-band collective mode at positive (negative) doping. It is also shown that our results differ markedly from the random phase approximation in the strong-coupling limit, even at high doping, while they compare favorably with existing quantum Monte Carlo numerical simulations.
\end{abstract}

Introduction. - Spin and charge excitation spectra of correlated fermionic systems may be conveniently accessed using Kotliar and Ruckenstein (KR) slave-boson representations of the microscopic model of interest [1] 9 . For instance, in the case of the Hubbard model on the square lattice, it has been recently shown that charge excitation spectra generically consist of a low-energy continuum, a zero-sound (ZS) collective mode, and another ' collective mode dispersing at energies scaling with the interaction strength [9. Hence these excitation spectra display the physics contained in the concepts introduced by Landau in his theory of the Fermi Liquid [10, and by Hubbard who established the splitting of the band due to the Coulomb interaction [11] which can now be incorporated in a single calculation. While earlier attempts suffered from various drawbacks [1, 8 , the one-loop calculation of spin and charge susceptibilities was recently shown to comply with lowest order perturbation theory and particlehole symmetry [9, 12. What happens when the latter is broken? Hubbard-type models are thought to contain the low-energy physics of superconducting cuprates, the phase diagrams of which are not symmetric under a sign change of the doping. One way to break the particle-hole symmetry is to take into account the next-nearest-neighbor (NNN) hopping. The purpose of this work is precisely to establish the influence of the latter on the charge excitation spectra, with parameter values generally accepted for the cuprates. We focus on the paramagnetic phase, free of symmetry breaking, in the thermodynamical limit and we resolve the full momentum dependence of the spectra. Owing to their weak temperature dependence, and to the relatively low magnetic-instability temperature $\left(T_{\mathrm{inst}} \approx t / 6\right)$, our results essentially apply to the entire phase diagram.

We perform our investigations within the KR slaveboson representation, which is able to capture interaction effects beyond the physics of Slater determinants. This approach reproduces the Gutzwiller approximation on the saddle-point level 13, which harbors the interaction driven Brinkman-Rice metal-to-insulator transition [14. Many valuable results have been obtained with KR [13] and related slave-boson representations [1,6]. For example the anti-ferromagnetic [15], spiral [16 19], and striped 20 24] phases have been described with these methods, as well as the competition between the latter two 24. Furthermore, it has been shown that the spiral order continuously evolves to the ferromagnetic order in the large $U$ regime $(U \gtrsim 60 t)$ 19. Consistently, in the two-band model, ferromagnetic instabilities were found in the doped Mott insulating regime only 25. Yet, ferromag- 
netic instability lines arise in the intermediate-coupling regime either through the introduction of a ferromagnetic exchange coupling [26], or due to a sufficiently large NNN hopping amplitude [27, or on the fcc lattice [28]. The framework has been used most recently to address strong correlation effects in the plates of a capacitor and a possible capacitance gain 29]. Furthermore, the comparison of groundstate energies to existing numerical simulations on the square lattice showed that the difference between the numerical estimate and the slave-boson result is less than $3 \%$ for $U=4 t[16$. For larger values of $U$ and doping larger than $15 \%$, it has been obtained that the slave-boson groundstate energy exceeds the exact diagonalization data by less than $4 \%$ for $U=8 t$, and less than $7 \%$ for $U=20 t$. The discrepancy increases when the doping is lowered [17]. In addition, quantitative agreement to quantum Monte Carlo (QMC) charge structure factors was established [8]. Furthermore, it has been shown in [9] that time-dependent Gutzwiller approximation and slave boson calculations at the Gaussian level exhibit both qualitative and quantitative differences, despite the exact correspondence between the Gutzwiller approximation and the saddle-point approximation to the KR representation.

The letter is organized as follows. Firstly we give a brief presentation of the spin-rotation-invariant (SRI) KR slave-boson representation of the Hubbard model and the method used to calculate dynamical response functions (more details can be found in, e.g., review 30). Then we evaluate the charge susceptibility from fluctuations captured within the one-loop approximation, and investigate the dispersion of its collective modes. Lastly we summarize the letter in the conclusion.

Model and method. - Within the SRI KR slaveboson representation 16 13 60 the Hubbard Hamiltonian is expressed as

$$
H=\sum_{i, j} t_{i j} \sum_{\sigma, \sigma^{\prime}, \sigma^{\prime \prime}} z_{i \sigma^{\prime \prime} \sigma}^{\dagger} f_{i \sigma}^{\dagger} f_{j \sigma^{\prime}} z_{j \sigma^{\prime} \sigma^{\prime \prime}}+U \sum_{i} d_{i}^{\dagger} d_{i}
$$

with auxiliary-boson operators $e_{i}, p_{i \mu}, d_{i}$ (for atomic states with respectively zero, single and double occupancy) and pseudo-fermion operators $f_{i \sigma}$. Note that in the approach, the on-site Coulomb interaction is represented by a term bilinear in bosonic operators. Yet this is at the expense of the hopping term, which is supplemented by the occupancy-change operator $z_{i \sigma \sigma^{\prime}}$. In order to preserve spin rotation symmetry the latter is defined as

$$
\underline{z}_{i}=e_{i}^{\dagger} \underline{L}_{i} M_{i} \underline{R}_{i} \underline{p}_{i}+\underline{\tilde{p}}_{i}^{\dagger} \underline{R}_{i} M_{i} \underline{L}_{i} d_{i}
$$

with

$$
\begin{aligned}
M_{i} & =\left[1+e_{i}^{\dagger} e_{i}+\sum_{\mu=0}^{3} p_{i \mu}^{\dagger} p_{i \mu}+d_{i}^{\dagger} d_{i}\right]^{1 / 2}, \\
\underline{L}_{i} & =\left[\left(1-d_{i}^{\dagger} d_{i}\right) \underline{\tau}^{0}-2 \underline{p}_{i}^{\dagger} \underline{p}_{i}\right]^{-1 / 2}, \\
\underline{R}_{i} & =\left[\left(1-e_{i}^{\dagger} e_{i}\right) \underline{\tau}^{0}-2 \underline{\tilde{p}}_{i}^{\dagger} \underline{\tilde{p}}_{i}\right]^{-1 / 2},
\end{aligned}
$$

and the $2 \times 2$ matrices in spin space $\underline{p}_{i}=\frac{1}{2} \sum_{\mu=0}^{3} p_{i \mu} \underline{\tau}^{\mu}$ and $\underline{\tilde{p}}_{i}=\frac{1}{2}\left(p_{i 0} \underline{\tau}^{0}-\mathbf{p}_{i} \cdot \underline{\tau}\right)$, which are built from the canonical operators $p_{i \mu}$, the identity matrix $\underline{\tau}^{0}$, and the Pauli matrices (for more details, see [30]).

Furthermore the auxiliary-boson operators generate a Fock space that contains more states than the physical ones. By definition the latter have exactly one atomic state per site, which means they belong to the subspace where, on each site $i$, the operator equality

$$
e_{i}^{\dagger} e_{i}+\sum_{\mu=0}^{3} p_{i \mu}^{\dagger} p_{i \mu}+d_{i}^{\dagger} d_{i}=1
$$

is satisfied. They additionally comply with the constraints

$$
\begin{gathered}
\sum_{\mu=0}^{3} p_{i \mu}^{\dagger} p_{i \mu}+2 d_{i}^{\dagger} d_{i}=\sum_{\sigma} f_{i \sigma}^{\dagger} f_{i \sigma}, \\
p_{i 0}^{\dagger} \mathbf{p}_{i}+\mathbf{p}_{i}^{\dagger} p_{i 0}-\mathrm{i} \mathbf{p}_{i}^{\dagger} \times \mathbf{p}_{i}=\sum_{\sigma, \sigma^{\prime}} \boldsymbol{\tau}_{\sigma \sigma^{\prime}} f_{i \sigma^{\prime}}^{\dagger} f_{i \sigma},
\end{gathered}
$$

which equate the number of fermions to the number of $p$ and $d$ bosons. When calculating the partition function as a functional integral [4,8, the physical constraints are then enforced with Lagrange multipliers $\alpha_{i}$ and $\beta_{i \mu}$. The internal gauge symmetry of the representation allows to gauge away the phases of $e_{i}$ and $p_{i \mu}$ by promoting the Lagrange multipliers to time-dependent fields $[6$, leaving us with radial slave-boson fields 31. Their saddle-point values may be viewed as an approximation to their exact expectation values that are generically non-vanishing [32]. However, the slave-boson field corresponding to double occupancy $d_{i}=d_{i}^{\prime}+\mathrm{i} d_{i}^{\prime \prime}$ has to remain complex [5, 6, 33.

Within the saddle-point approximation, the quasiparticle mass is divided by a factor $z_{0}^{2}$, which also plays the role of a quasiparticle residue. For the paramagnetic solution

$$
z_{0}^{2}=\frac{2 p_{0}^{2}(e+d)^{2}}{1-\delta^{2}}
$$

where $e, p_{0}$, and $d$ are the saddle-point values of the boson fields, and $\delta=1-n$ is the hole doping from half-filling. The quasiparticle dispersion is renormalized as

$$
E_{\mathbf{k}}=z_{0}^{2} t_{\mathbf{k}}-\left(\mu-\beta_{0}\right)
$$

with $\mu$ the chemical potential, and $\beta_{0}$ the saddle-point value of the Lagrange multiplier enforcing the constraint (5a). The bare quasiparticle energy is

$$
t_{\mathbf{k}}=-2 t\left(\cos k_{x}+\cos k_{y}\right)-4 t^{\prime} \cos k_{x} \cos k_{y}
$$

on the square lattice, when hopping processes of amplitude $t_{i j}=-t$ between nearest-neighbor sites and $t_{i j}=-t^{\prime}$ between NNN ones are taken into account. In the absence of the latter, the energy and the velocity $v_{\mathbf{k}}=\left|\partial t_{\mathbf{k}} / \partial \mathbf{k}\right|$ are symmetric with respect to the Fermi level at half-filling $n=1$ (see the upper panel of Fig. 11). The quasi-parabolic 


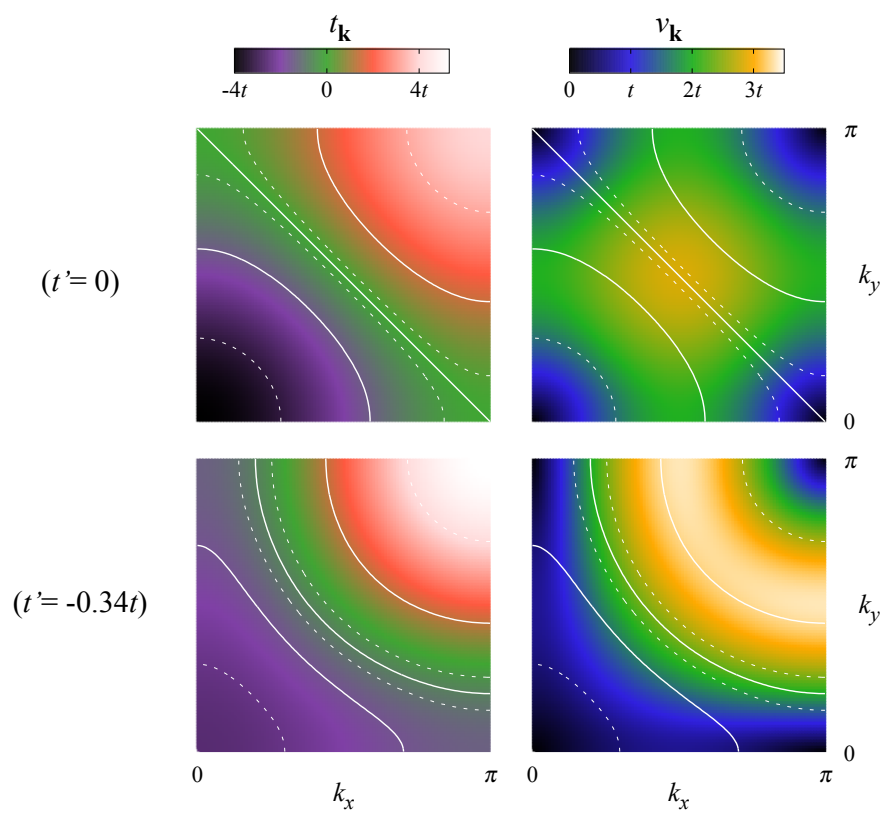

Fig. 1: (Color online) Bare quasiparticle energy $t_{\mathbf{k}}$ and velocity $v_{\mathbf{k}}$ on the square lattice for $t^{\prime}=0$ and $-0.34 t$. The white lines are the Fermi surface plotted for density values $n=0.125,0.5$, $0.875,1,1.125,1.5$, and 1.875 (from the lower left to the upper right corner of each map).

dispersion in the vicinity of the k-points $\Gamma=(0,0)$ and $\mathrm{M}=(\pi, \pi)$ results in a nearly circular Fermi surface at large doping $|1-n| \approx 1$. The lower panel of Fig. 1 1 shows that adding a finite hopping between NNN breaks the doping-reversal symmetry of the dispersion. A negative $t^{\prime}$ (for $t>0$ ) non-uniformly increases the energy and enhances the velocity, as well as the isotropy of the dispersion, around $\mathrm{M}$ at the expense of the vicinity of $\Gamma$. Hence the Fermi velocity is quasi-isotropic at the density $n>1$ for $t^{\prime}=-0.34 t$.

The saddle-point approximation is exact in the large degeneracy limit, while the Gaussian fluctuations are of order $1 / N$ [6. In addition it obeys a variational principle in the limit of large spatial dimensions where the Gutzwiller approximation becomes exact for the Gutzwiller wave function [34. Within the Gaussian fluctuation approximation, the action is expanded to second order in field fluctuations

$$
\begin{gathered}
\psi(k)=\left(\delta e(k), \delta d^{\prime}(k), \delta d^{\prime \prime}(k), \delta p_{0}(k), \delta \beta_{0}(k), \delta \alpha(k),\right. \\
\left.\delta p_{1}(k), \delta \beta_{1}(k), \delta p_{2}(k), \delta \beta_{2}(k), \delta p_{3}(k), \delta \beta_{3}(k)\right)
\end{gathered}
$$

around the paramagnetic saddle-point solution $\psi_{\mathrm{MF}}=$ $\left(e, d, 0, p_{0}, \beta_{0}, \alpha, 0,0,0,0,0,0\right)$ as

$$
\int d \tau \mathcal{L}(\tau)=\mathcal{S}_{\mathrm{MF}}+\sum_{k, \mu, \nu} \psi_{\mu}(-k) S_{\mu \nu}(k) \psi_{\nu}(k)
$$

(the matrix $S$ is given in Ref. 9]). Here $k=\left(\mathbf{k}, \nu_{n}\right)$ with the bosonic Matsubara frequency $\nu_{n}=2 \pi n T$, and $\sum_{k}=T \sum_{\nu_{n}} L^{-1} \sum_{\mathbf{k}}$ with $L$ the number of lattice sites.
The correlation functions of boson fields are then Gaussian integrals which can be obtained from the inverse of the fluctuation matrix $S$ as $\left\langle\psi_{\mu}(-k) \psi_{\nu}(k)\right\rangle=\frac{1}{2} S_{\mu \nu}^{-1}(k)$. Using the density fluctuation $\delta \mathcal{N}=\delta\left(d^{\dagger} d-e^{\dagger} e\right)$, the charge susceptibility is

$$
\begin{aligned}
\chi_{c}(k) & =\langle\delta \mathcal{N}(-k) \delta \mathcal{N}(k)\rangle \\
& =2 e^{2} S_{1,1}^{-1}(k)-4 e d S_{1,2}^{-1}(k)+2 d^{2} S_{2,2}^{-1}(k) .
\end{aligned}
$$

The dynamical response function is eventually obtained within the analytical continuation $\mathrm{i} \nu_{n} \rightarrow \omega+\mathrm{i}^{+}$. In the present work, for temperature $T=t / 100$, the infinitesimal imaginary part is set between $10^{-6} t$ and $10^{-4} t$, depending on the needed frequency resolution.

The evaluation of the correlation functions in the paramagnetic state 3 , 4, 7, 8, yields the charge dynamical response function 9]

$$
\chi_{c}(k)=\frac{A(k)+B(k)\left(\omega+\mathrm{i} 0^{+}\right)^{2}}{C(k)+D(k)\left(\omega+\mathrm{i} 0^{+}\right)^{2}}
$$

where

$$
\begin{aligned}
A(k) & =\tilde{S}_{33}\left[2 p_{0}^{2} \Gamma_{1}(k)-8 d p_{0} \Gamma_{2}(k)+8 d^{2} \Gamma_{3}(k)\right] \\
B(k) & =2 e d p_{0}^{2} S_{55}(k) \\
C(k) & =\tilde{S}_{33}\left[\Gamma_{2}^{2}(k)-\Gamma_{1}(k) \Gamma_{3}(k)\right] / e^{2} S_{55}(k), \\
D(k) & =-\frac{d\left[p_{0}^{2} \Gamma_{1}(k)+2(e-d) p_{0} \Gamma_{2}(k)+(e-d)^{2} \Gamma_{3}(k)\right]}{e(e+d)^{2}}
\end{aligned}
$$

with

$$
\begin{aligned}
\Gamma_{1}(k)= & -S_{55}(k)\left[e^{2} S_{22}(k)-2 e d S_{12}(k)+d^{2} S_{11}(k)\right] \\
& +\left[e S_{25}(k)-d S_{15}(k)\right]^{2} \\
\Gamma_{2}(k)= & -S_{55}(k)\left[e^{2} S_{24}(k)-p_{0} e S_{12}(k)-e d S_{14}(k)\right. \\
& \left.+d p_{0} S_{11}(k)\right]+\left[e S_{25}(k)-d S_{15}(k)\right] \\
& {\left[e S_{45}(k)-p_{0} S_{15}(k)\right], } \\
\Gamma_{3}(k)= & -S_{55}(k)\left[e^{2} S_{44}(k)-2 e p_{0} S_{14}(k)+p_{0}^{2} S_{11}(k)\right] \\
& +\left[e S_{45}(k)-p_{0} S_{15}(k)\right]^{2} \\
\tilde{S}_{33}= & -\frac{2 p_{0}^{2}}{1-\delta^{2}} \varepsilon_{0} .
\end{aligned}
$$

Here the semi-renormalized kinetic energy is

$$
\varepsilon_{0}=\frac{2}{L} \sum_{\mathbf{k}} t_{\mathbf{k}} n_{F}\left(E_{\mathbf{k}}\right)
$$

with the Fermi function $n_{F}(\epsilon)=1 /(\exp (\epsilon / T)+1)$.

Charge collective modes. - The lengthy but straightforward expansion of the terms (13) shows that they are invariant under the reversal of the doping sign when $t^{\prime}=0$, so that $\chi_{c}(k)$ is symmetric 12. This result stems from the particle-hole symmetry of the Hubbard model on a bipartite lattice, which pervades the dispersion and the susceptibility of the quasiparticles, as well as the paramagnetic saddle-point solution of the boson fields. 


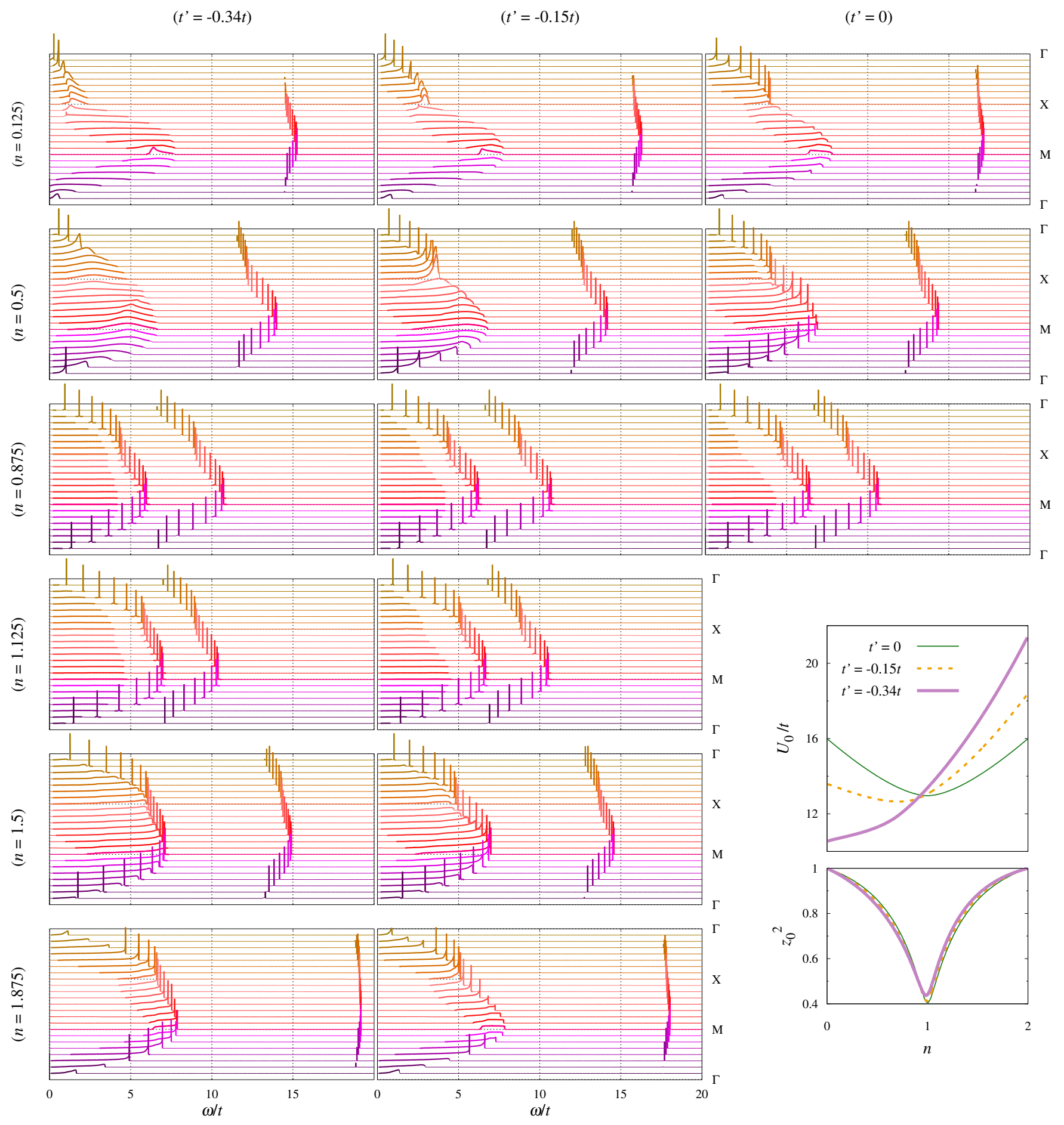

Fig. 2: (Color online) The imaginary part of the charge response function $\chi_{c}(k)$, the coupling scale $U_{0}$, and the mass renormalization factor $z_{0}^{2}$ for different values of NNN hopping $t^{\prime}$ and density $n$. The spectrum of $\operatorname{Im} \chi_{c}(k)$ is plotted for momenta along the path linking $\Gamma=(0,0), \mathrm{X}=(\pi, 0)$ and $\mathrm{M}=(\pi, \pi)$. Parameters: $U=10 t, T=t / 100$. 
However the symmetry does not hold for a finite $t^{\prime}$. As an illustration, the inelastic charge response given by $\operatorname{Im} \chi_{c}(k)$ is plotted for $t^{\prime}=0,-0.15 t$, and $-0.34 t$ at different densities in Fig. 2 Since for $t^{\prime}=0$ the charge susceptibility is symmetric, it is shown only at densities $n<1$.

The spectra are composed of a broad continuum generated by incoherent single-particle-hole excitations, and the peaks of two collective modes above it. Both collective excitations have their minimum at $\Gamma$ and their maximum at $\mathrm{M}$. For the large coupling value $U=10 t$, the correlation effects are important around half-filling where the continuum width is scaled down by the factor $z_{0}^{2}$ and a large portion of its intensity is transferred to the peaks. This prediction compares favorably with QMC simulations [35], within the available energy resolution. Due to the numerical approximations inherent to the method and the high temperature $(T=t / 3)$ at which simulations are performed, the QMC spectra are Gaussian-like distributions. At low doping $(|\delta| \approx 0.05)$ nearly all the intensity of the $\mathrm{QMC}$ signal is located at high energy, with the maximum between the positions of the two collective modes found within the slave-boson approach. With increasing the doping the bell of the QMC spectrum moves at a lower energy, although it retains a high-energy tail. In the slave-boson response, this corresponds to an increase of the continuum intensity, which also merges with the lower-energy collective mode. At the same time, the higher-energy peak goes up while its weight decreases. However, we shall note that the paramagnetic slave-boson result does not match the QMC response at half-filling. This should not be surprising since the QMC ground-state is antiferromagnetic at zero doping [36].

The NNN hopping term visibly modifies the shape of the continuum since the bare quasiparticle dispersion $t_{\mathbf{k}}$ is changed. However $t^{\prime}$ only has a small influence on the renormalization factor $z_{0}^{2}$, as shown in the lower right corner of Fig. 2. Increasing $\left|t^{\prime}\right|$ slightly narrows down (widens) the continuum for hole (particle) doping. The variation is a little more noticeable in the vicinity of $n=1$ where the width change is more important. This is in agreement with exact diagonalization results [37, 38]. In contrast, as discussed below in detail, the effect of varying $t^{\prime}$ is more visible on the dispersion of the collective modes.

The higher-energy mode follows from the upperHubbard-band (UHB) with its excitation energy given in the strong coupling limit $\left(U \gg U_{0}\right)$ by $\omega_{\mathrm{UHB}}(\mathbf{k}) \approx$ $U \sqrt{1-\frac{U_{0}}{2 U}\left(1-3|\delta|+(1-|\delta|) \frac{\varepsilon_{\mathbf{k}}}{\varepsilon_{0}}\right)}$, which increases as the Coulomb coupling $U$, 9 . The coupling scale

$$
U_{0}=-\frac{8 \varepsilon_{0}}{1-\delta^{2}}
$$

is plotted in Fig. 2, and

$$
\varepsilon_{\mathbf{k}}=\frac{2}{L} \sum_{\mathbf{q}} t_{\mathbf{q}+\mathbf{k}} n_{F}\left(E_{\mathbf{q}}\right) .
$$

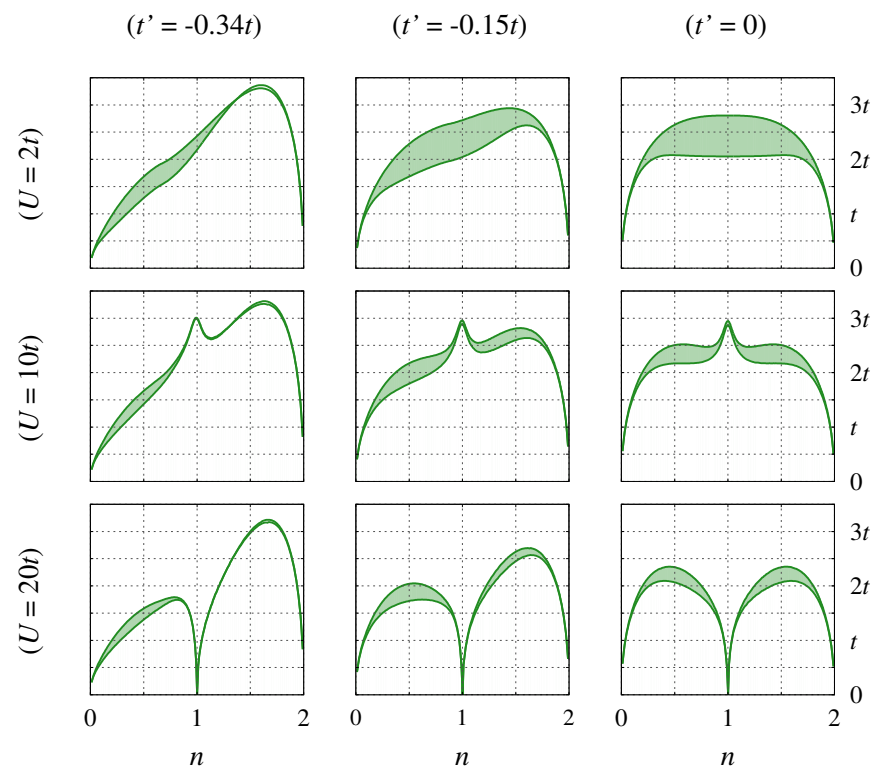

Fig. 3: (Color online) Dispersion of the zero-sound velocity as a function of the density, for different values of $t^{\prime}$ and $U$. Parameter: $T=t / 100$.

In this regime the mode starts around the energy $U+$ $U_{0}(|\delta|-1 / 2)$ and extends over a range $\approx(1-|\delta|) U_{0} / 2$. In the opposite limit $\left(U \ll U_{0}\right)$ the dispersion is $\omega_{\mathrm{UHB}}(\mathbf{k}) \approx$ $\frac{U_{0}}{2} \sqrt{1+\frac{U}{2 U_{0}}\left(1+7 \delta^{2}-\left(1-\delta^{2}\right) \frac{\varepsilon_{\mathbf{k}}}{\varepsilon_{0}}\right)}$. Although the weakcoupling expression is a rough approximation for the coupling value $U=10 t$, it nevertheless yields the qualitative behavior of the mode. It locates the bottom of its dispersion around $U_{0} / 2+U \delta^{2}$, and gives a width of about $U\left(1-\delta^{2}\right) / 4$. As illustrated in Fig. 2, doping the system increases the collective-excitation energy and narrows down its dispersion. The comparison of the spectra at a fixed doping shows that $t^{\prime}$ softens (hardens) the UHB mode for large positive (negative) values of doping. Indeed a negative $t^{\prime} / t$ flattens the quasiparticle dispersion around $\Gamma$ while it increases their velocity around M (see Fig. 1). As a result, the minimum of the average kinetic energy $\varepsilon_{0}$ is moved from $n=1$ for $t^{\prime}=0$ to $n>1$ for $t^{\prime} / t<0$. This reduces $U_{0}$ at $n<1$ and increases it at $n>1$ (see Fig. 2).

The lower-energy collective excitation, called the ZS mode, is located between the upper edge of the continuum $\omega_{\text {cont }}(\mathbf{k})$ and the UHB mode. Contrary to the UHB mode, its energy vanishes at $\Gamma$ as its dispersion is linear at long wavelength. In this limit one then defines the ZS velocity as $c_{s}(\hat{\mathbf{k}})=\omega_{\mathrm{ZS}}(\mathbf{k}) /|\mathbf{k}|$. It is anisotropic on the square lattice, with the minimum in the $\mathrm{X}$-direction and the maximum in the M-direction. Fig. 3 shows the density dependence of its dispersion for different values of coupling and NNN hopping amplitude. Strong correlations increase the ZS velocity around half filling for $U$ smaller than the critical value $U_{c}=2(8 / \pi)^{2} t \approx 12.97 t$. Above the critical coupling, $c_{s}(\hat{\mathbf{k}})$ vanishes at half filling as the state is insulating. For $t^{\prime}=0$ the density dependence is 
$(n=0.5)$

$$
\begin{gathered}
\operatorname{Re} f^{s} \\
10^{3} \\
15 \\
0 \\
-15 \\
-10^{3}
\end{gathered}
$$

$\operatorname{Im} \chi_{\mathrm{c}}$
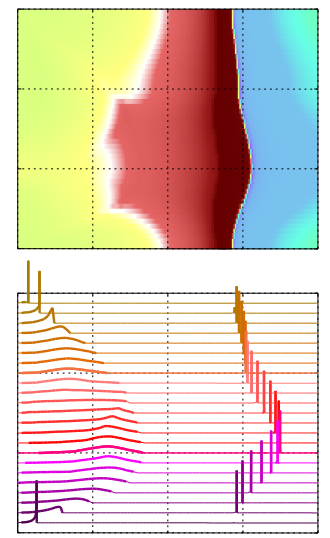

$\operatorname{Im} \chi_{\mathrm{RPA}}$

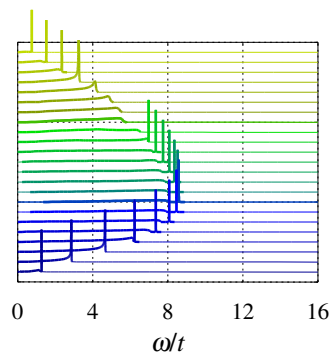

$(n=1.5)$

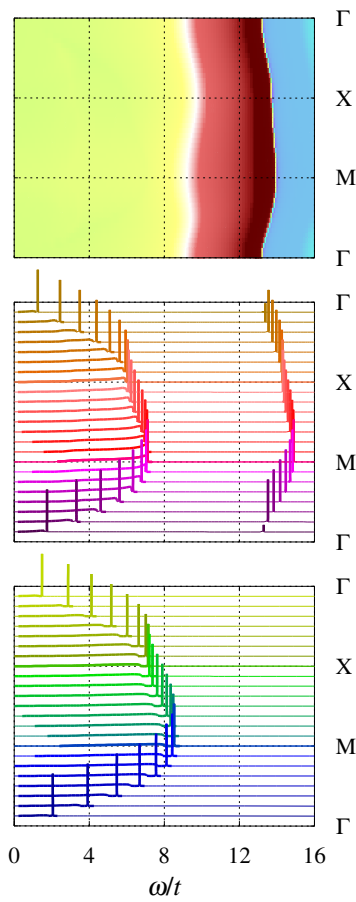

Fig. 4: (Color online) Energy and momentum dependence of $\operatorname{Re} f^{s}(k)$, and comparison between the slave-boson charge response $\chi_{c}(k)$ and the RPA result $\chi_{\mathrm{RPA}}(k)$ for density $n=0.5$ and 1.5. Parameters: $t^{\prime}=-0.34 t, U=10 t, T=t / 100$.

symmetric from either side of $n=1$. Increasing the amplitude of $t^{\prime}$ decreases (increases) the velocity at positive (negative) doping, in accord with the modification of the bare quasiparticle dispersion $t_{\mathbf{k}}$. As previously noted for the Fermi velocity, the anisotropy of $c_{s}(\hat{\mathbf{k}})$ is reduced at negative doping by the NNN hopping.

Fig. 2 shows that for $t^{\prime}=0$ a large doping suppresses the ZS mode in a large part of the Brillouin zone. For a finite $t^{\prime}$, a positive doping enhances the inhibition, while a negative doping favors the ZS mode. The change of the quasiparticle dispersion is not the sole cause of the ZS mode damping. There is also a dynamical screening of the interaction induced by correlations. In order to discuss this effect, we write the charge response function as

$$
\chi_{c}(k)=\frac{\chi_{0}(k)}{1+f^{s}(k) \chi_{0}(k)}
$$

with the Lindhard function

$$
\chi_{0}(k)=\frac{2}{L} \sum_{\mathbf{q}} \frac{n_{F}\left(E_{\mathbf{q}+\mathbf{k}}\right)-n_{F}\left(E_{\mathbf{q}}\right)}{\left(\omega+\mathrm{i} 0^{+}\right)-\left(E_{\mathbf{q}+\mathbf{k}}-E_{\mathbf{q}}\right)} .
$$

Here $f^{s}(k)=\chi_{c}(k)^{-1}-\chi_{0}(k)^{-1}$ represents an effective interaction that reduces to $U / 2$ in the weak-coupling limit. There, the random-phase approximation (RPA) result

$$
\chi_{\mathrm{RPA}}(k)=\frac{\chi_{0}^{(0)}(k)}{1+\frac{U}{2} \chi_{0}^{(0)}(k)}
$$

is recovered [9]. Since $\chi_{0}(\mathbf{k})$ is real above $\omega_{\text {cont }}(\mathbf{k})$ and has a negative value, this explains how the pole associated to the ZS mode appears just beyond the continuum upper edge when increasing $U$. However, as one can expect, the RPA perturbation approach breaks down at large coupling. Fig. 4 shows the strong dependence on momentum and frequency of the complex function $f^{s}(k)$ for $U=10 t$. Its value, indeed, can drastically differ from $U / 2$. It even goes to infinity at an energy $\omega \sim U$, which gives rise to the pole of the UHB mode. The RPA response neither possesses the higher-energy mode, nor accounts for the renormalization of the continuum width. Going back to the causes of the ZS mode suppression, one can note that $\chi_{\mathrm{RPA}}(k)$ has no peak around X at density $n=0.5$, contrary to $n=1.5$. The damping here is only ascribed to the differences in the quasiparticle dispersion. However in the slave-boson response at $n=0.5$, the suppression extends up to a larger region of the $\mathbf{k}$-space. The enhancement of the damping stems from the screening of the effective potential $f^{s}(k)$ which even turns it negative around M. In contrast, for density $n=1.5$, the screening is limited to higher energies and does not prevent the ZS mode. As recently revealed by the detailed comparison 35 between QMC simulations and the RPA, strong correlations persist in multiparticle responses over doping values larger than the one for single-particle properties, especially in the charge channel. In agreement with the QMC results, the slave-boson charge response at low density $n \lesssim 0.5$ is essentially formed by a broad featureless continuum in a large part of the Brillouin zone, in contrast to the RPA intensity which shows a peaked maximum at the continuum upper edge. The latter results from a resonance with the ZS mode, and forms a well separated peak at low temperature. The RPA pole is present around $\mathbf{k}=\mathrm{M}$ even at density as low as $n=0.1$, while it is suppressed by correlations in the slave-boson response.

Conclusion. - We have calculated the charge excitation spectra of the $t-t^{\prime}-U$ Hubbard model on the square lattice in the thermodynamical limit within KR slave boson formalism. While our approach reduces to RPA in the weak coupling regime, and while both approaches may be used in the thermodynamical limit to resolve the full momentum dependence of the spectra, they exhibit significant differences from the intermediate coupling regime on. In particular, the former is sensitive to the renormalization of the continuum width and displays a higher-energy mode originating from the upper Hubbard band. Furthermore sharp peaks artificially predicted by the RPA that are absent in QMC simulations are fully damped in our approach, too. In fact, a good agreement between our paramagnetic calculations and QMC simulations has been found, provided the latter are, of course, performed outside a magnetic phase. The influence of $t^{\prime}$ on the shape of the response continuum is strongest for large doping, though the correlation-induced renormalization of its width is barely affected. Increasing $-t^{\prime}$ softens 
(hardens) the high-frequency UHB collective mode at positive (negative) doping. When approaching half-filling, the mode loses most of its $t^{\prime}$ dependence while it gets broader, and is shifted to lower frequency. For low (high) density $n \lesssim 0.5(n \gtrsim 1.5)$, the suppression of the ZS collective mode is favored (reduced) by $t^{\prime}$. Its damping is enhanced by a dynamical screening of the interaction most effective for large momenta. The averaged ZS velocity decreases (increases) with increasing $-t^{\prime}$ for hole (particle) doping, following the change in the quasiparticle dispersion. The above discussed collective modes should be observable in the paramagnetic phase of the superconducting cuprates by means of inelastic neutron scattering experiments. This, in fact, could even be a way to measure $t^{\prime}$.

\section{$* * *$}

We gratefully thank T. Kopp for several stimulating discussions. The authors acknowledge the financial support of the French Agence Nationale de la Recherche (ANR), through the program Investissements d'Avenir (ANR-10LABX-09-01), LabEx EMC3.

\section{REFERENCES}

[1] LI T. C., WÖLFLE P. and HIRSCHFELD P. J., Phys. Rev. B, 40 (1989) 6817.

[2] RASUL J. W., LI T. C., J. Phys. C, 21 (1988) 5119; LI T. C. and RASUL J. W., Phys. Rev. B, 39 (1989) 4630; RASUL J. W., LI T. C., and BECK H., Phys. Rev. B, 39 (1989) 4191.

[3] LAVAGna M., Phys. Rev. B, 41 (1990) 142.

[4] LI T. C., SUN Y. S., and WÖLfLE P., Z. Phys. B, 82 (1991) 369.

[5] BANG Y., CASTELLANI C., GRILLI M., KOTLIAR G., RAIMONDI R., and WANG Z., Int. J. of Mod. Phys. B, 6 (1992) 531.

[6] FRÉSARD R. and P. WÖLFLE, Int. J. of Mod. Phys. B, 6 (1992) 685; 6 (1992) 3087.

[7] LI T. C. and BÉNARD P., Phys. Rev. B, 50 (1994) 17837.

[8] ZIMMERMANN W., FRÉSARD R., and WÖLFLE P., Phys. Rev. B, 56 (1997) 10097.

[9] DAO V. H. and FRÉSARD R., Phys. Rev. B, 95 (2017) 165127.

[10] LANDAU L. D., Sov. Phys. JETP, 3 (1956) 920.

[11] HubBard J., Proc. Roy. Soc. London A, 276 (1963) 238; 281 (1964) 401.

[12] DAO V. H. and FRÉSARD R., arXiv:1708.07760 preprint, 2017.

[13] KOtliar G. and RUCKenstein A. E., Phys. Rev. Lett., 57 (1986) 1362.

[14] BRINKMAN W. F. and RICE T. M., Phys. Rev. B, 2 (1970) 4302.

[15] Lilly L., MURamatsu A., and Hanke W., Phys. Rev. Lett., 65 (1990) 1379.

[16] FRÉSARD R., DZIERZAWA M., and P. WÖLFLE, Europhys. Lett., 15 (1991) 325.

[17] FRÉSARD R. and WÖLFLE P., J. Phys.: Condens. Matter, 4 (1992) 3625.
[18] IGOSHEV P. A., TIMIRGAZIN M. A., ARZHNIKOV A. K. and IRKHIN V. Y., JETP Lett., 98 (2013) 150.

[19] MÖLlER B., DOLL K., and FRÉSARD R., J. Phys.: Condensed Matter, 5 (1993) 4847.

[20] SEIBOLD G., SIGMUND E., and HIZHNYAKOV V., Phys. Rev. B, 57 (1998) 6937; SEIBOLD G. and LORENZANA J., Phys. Rev. Lett., 86 (2001) 2605.

[21] FLECK M., LICHTENSTEIN A. I., and OLEŚ A. M., Phys. Rev. B, 64 (2001) 134528.

[22] LOREnZANA J. and SEIBOLD G., Phys. Rev. Lett., 89 (2002) 136401; 90 (2003) 066404; 94 (2005) 107006.

[23] RACZKOWSKI M., FRÉSARD R., and OLEŚS A. M., Phys. Rev. B, 73 (2006) 174525; RACZKOWSKI M., et al. Phys. Rev. B, 76 (2007) 140505(R).

[24] RACZKOWSKI M., FRÉSARD R., and OLEŚ A. M., Europhys. Lett., 76 (2006) 128.

[25] FRÉSARD R. and LAMBOLEY M., J. Low Temp. Phys., 126 (2002) 1091.

[26] LHOUTELliER G., FRÉSARD R., and OLEŚ A. M., Phys. Rev. B, 91 (2015) 224410.

[27] FRÉSARD R. and ZIMMERMANN W., Phys. Rev. B, 58 (1998) 15288.

[28] IGOSHEV P.A., TIMIRGAZIN M.A., GILMUTDINOV V.F., ARZHNIKOV A.K., and IRKHIN V.YU., J. Phys: Condens. Matter, 27 (2015) 446002.

[29] STEFFEn K., FRÉSARD R., and KOPP T., Phys. Rev. $B, 95$ (2017) 035143.

[30] FRÉSARD R., KROHA J., and P. WÖLFLE, in Theoretical Methods for Strongly Correlated Systems, edited by A. Avella and F. Mancini, Springer Series in Solid-State Sciences, Vol. 171 (Springer-Verlag, Berlin) 2012, pp. 65101.

[31] FRÉSARD R. and KOPP T., Nucl. Phys. B, 594 (2001) 769.

[32] FRÉSARD R., OUERDANE H., and KOPP T., Nucl. Phys. B, 785 (2007) 286.

[33] JOliCceur TH. and LE GUILlOU J. C., Phys. Rev. B, 44 (1991) 2403.

[34] METZner W. and VOLLHARDT D., Phys. Rev. Lett., 62 (1989) 324; Phys. Rev. B, 37 (1988) 7382; METZNER W., Z. Phys. B, 77 (1989) 253.

[35] KUNG Y. F., NOWADNICK E. A., JIA C. J., JOHNSTON S., MORITZ B., SCALETTAR R. T., and DEVEREAUX T. P., Phys. Rev. B, 92 (2015) 195108.

[36] HUANG E. W., MENDL C. B., JIANG H.-C., MORITZ B., and DEVEREAUX T. P., arXiv:1709.02398 preprint, 2017.

[37] JIA C. J., CHEN C.-C., SORINI A. P., MORITZ B., and DEVEREAUX T. P., New J. Phys., 14 (2012) 113038.

[38] in the Supplemental Material of WANG Y., JIA C. J., MORITZ B., and DEVEREAUX T. P., Phys. Rev. Lett., 112 (2014) 156402. 